\title{
Geometric Analysis of the Vibration of Rubber Wiper Blade
}

\author{
Tsai-Jung Chen and Ying-Ji Hong*
}

\begin{abstract}
The purpose of this paper is to work out the theoretical aspects of the vibration problem of rubber wiper blade on convex windshield. Over the past 20 years, some 2-dimensional spring-mass models were presented in engineering science to simulate the vibration of rubber wiper blade on windshield. In this paper, we will consider the elasticity perspective on this 3-dimensional vibration problem. Our theoretical analysis suggests that there should exist two classes of vibration frequencies corresponding to "*-exact deformations (Class I)" and "*-closed deformations (Class II)". We prove mathematical theorems on the characterization of deformations of Class I. We also explain how elementary deformations of Class II can be constructed. We then deduce two mathematical formulas, for the vibration problem of rubber wiper blade on convex windshield, from our theoretical analysis. Our theoretical predictions are in almost perfect agreement with experimental data. One of the crucial steps of our analysis is a decomposition theorem motivated by the de Rham Cohomology and the Hodge Theory.
\end{abstract}

\section{Introduction}

The purpose of this paper is to work out the theoretical aspects of the vibration problem of rubber wiper blade on convex windshield. This problem has been actively studied in engineering science for more than 20 years. See $3,6,18,20,28,29,31,33,37$. Generally, the vibration frequencies of rubber wiper blade on convex windshield are very complicated. Figure 1.1 shows a FFT (Fast Fourier Transform) diagram for the vibration frequencies of rubber wiper on convex windshield before reversal. On this FFT diagram, frequencies below $25,600 \mathrm{~Hz}$ can be trusted.

We try to understand the vibration frequencies of rubber wiper blade on convex windshield from the theoretical/mathematical physics point of view. Our theoretical analysis (Section 3) suggests that there should exist two classes of vibration frequencies corresponding to "*-exact deformations (Class I)" and "*-closed deformations (Class II)".

Received November 15, 2020; Accepted December 3, 2020.

Communicated by Mu-Tao Wang.

2020 Mathematics Subject Classification. 53C07, 70S05, 58A14, 35J05.

Key words and phrases. Hodge theory, wave equation, elasticity mechanics, rubber, vibration.

*Corresponding author. 


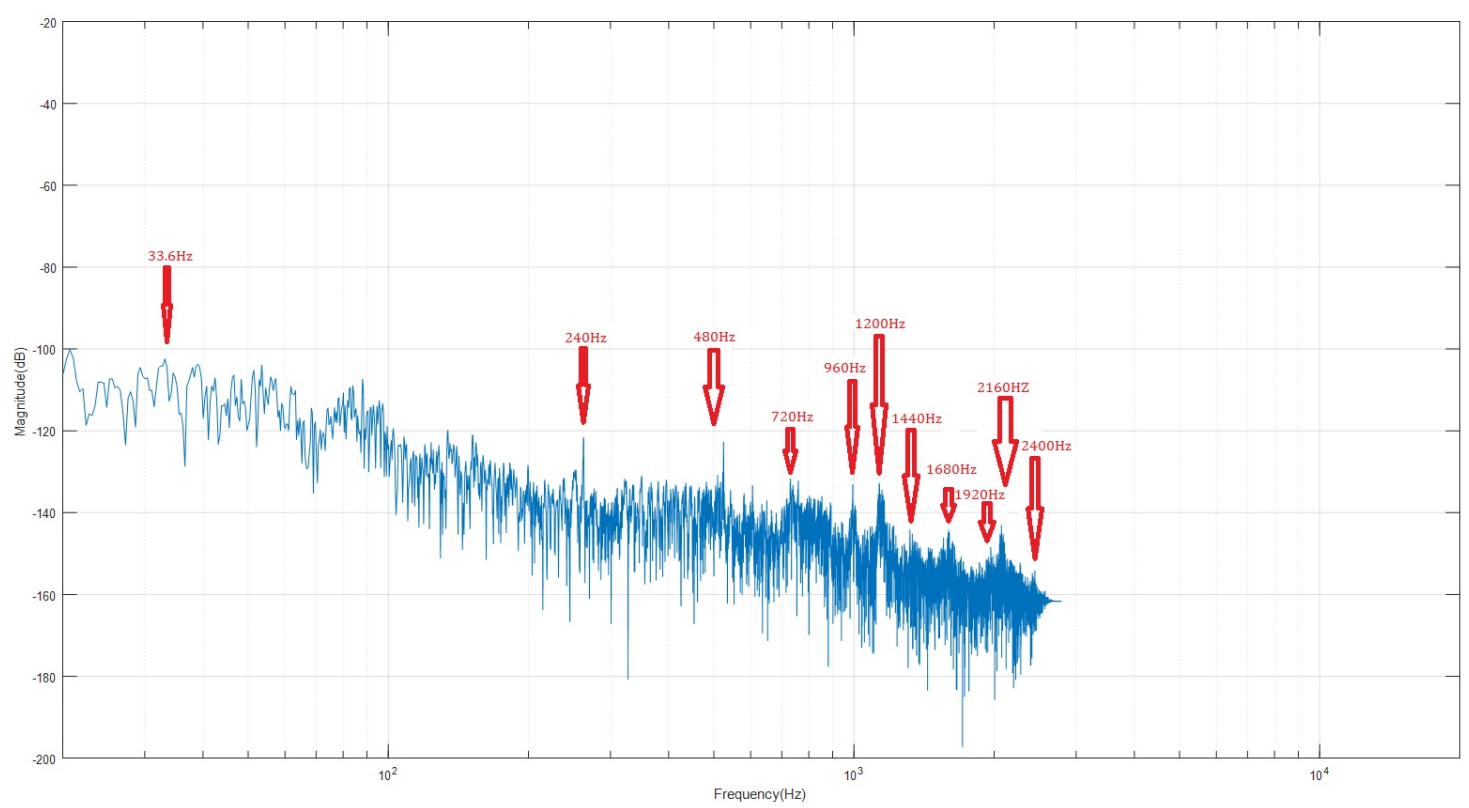

Figure 1.1: Vibration frequencies of rubber wiper blade on convex windshield.

Let $\rho \mathrm{kg} / \mathrm{m}^{3}$ denote the density of the rubber wiper. Let $l \mathrm{~m}$ denote the length of the rubber wiper. Then most of the vibration frequencies of Class I should locate around

$$
\sqrt{\frac{\lambda+2 \mu}{\rho}} \cdot \frac{n}{2 l} \mathrm{~Hz} \quad(\text { Class I) }
$$

where $n$ is a positive integer. Some of the vibration frequencies of Class II should appear around

$$
\sqrt{\frac{\mu}{\rho}} \cdot \frac{n}{2 l} \mathrm{~Hz} \quad \text { (Class II). }
$$

However, the vibration frequencies of Class II are loosely distributed, general low vibrationfrequencies of Class II may appear. Here $\lambda$ and $\mu$ are the "Lame coefficients". Lame coefficients are material constants of the rubber wiper. These material constants are related to the "Young modulus $E$ " and the "Poisson ratio $\sigma$ " as follows:

$$
\lambda=\frac{\sigma \cdot E}{(1+\sigma) \cdot(1-2 \sigma)} \quad \text { and } \quad \mu=\frac{E}{2 \cdot(1+\sigma)} .
$$

Experimental data has amazingly supported our theoretical predictions. In Figure 1.1. peaks of the specific vibration frequencies predicted by our mathematical formulas are indicated. In Figure 1.1, the strange vibration frequency around $510 \mathrm{~Hz}$, higher than the predicted frequency $480 \mathrm{~Hz}$, is caused by machine noise. For the details of comparison of experimental data with our mathematical formulas, see [10]. 
Somewhat surprisingly, one of the crucial steps of our analysis is a "decomposition theorem" (see Theorem 3.3) motivated by the de Rham Cohomology and the Hodge Theory. We will explain the physical aspects of this vibration problem in Section 2, Section 3 is devoted to the mathematical analysis of this vibration problem. To reduce the "wind resistance" on the windshield of a moving vehicle, the shape of windshield is generally strictly convex [7. This strict convexity of windshield leads to the boundary condition (3.9) for our mathematical formulation. Then in Section 4, we will explain how our analysis leads to our physical predictions on the vibration frequencies of rubber wiper blade on convex windshield. The mathematical analysis presented in this article is motivated by the authors previous work on differential geometry and theoretical physics $[22[26]$.

2. Physical aspects of the vibration problem

When a rubber wiper blade moves on the convex windshield of an automobile, there are 4 different forces acting on it: pressure on the wiper adapter, support force from the windshield, drag force from the wiper arm, and the frictional force acting on the rubber wiper blade. Figure 2.1 shows a 2-dimensional force diagram for the rubber wiper blade.

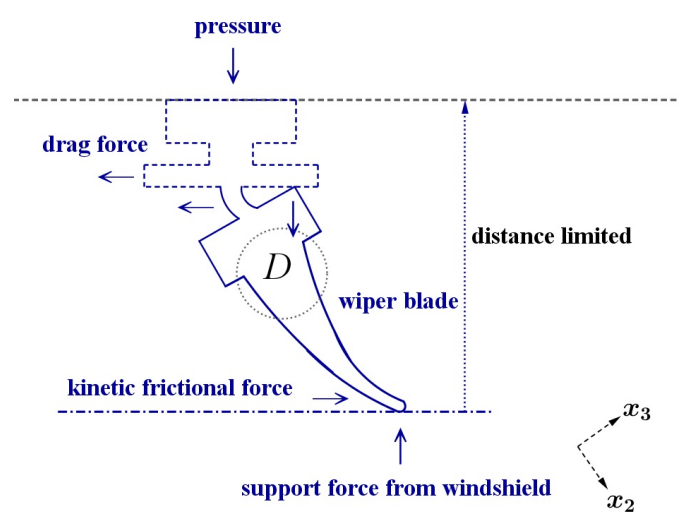

Figure 2.1: 2D Force Diagram.

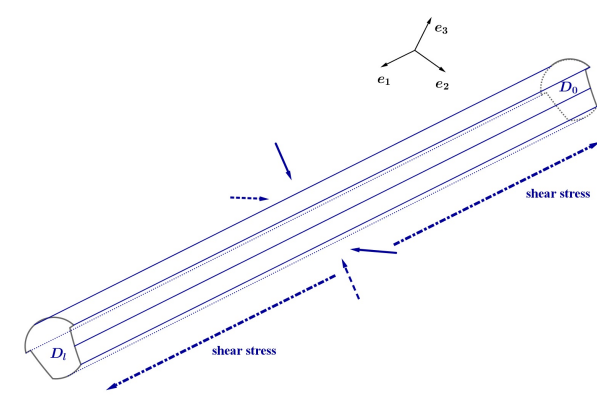

Figure 2.2: Slightly Deformed Region.

To keep rubber wiper blade working normally, the bending (deformation) of rubber wiper blade should not be severe to avoid wiper jumping or malfunctioning. Thus a suitable distance between the wiper adapter and the convex windshield must be retained. The wiper arm is always moving above the windshield with the pressure on the rubber wiper being applied by a spring under the wiper arm. 


\subsection{Hyperelasticity and viscoelasticity}

The rubber wiper blade is made of elastic material rubber. Here we explain the physical properties of rubber wiper briefly. Rubber is a polymer material consisting of very large molecules (macromolecules). These macromolecules are linked together by the Van der Waals force (see $[21,38,39]$ ). When rubber is deformed slightly, it behaves like a "hyperelastic" body [30]. Here "hyper-elastic" means that if we remove the force acting on a rubber, this rubber will return to its original shape and the "stored energy" in rubber will be released. Usually we consider the "stored energy" in rubber as a "potential function for deformation". See Appendix A of [16].

When rubber is deformed seriously, part of the linking between macromolecules will be broken. In this case, this rubber will not be able to return to its original structure. This phenomenon is called "viscoelasticity". In this case, the "conservation law of mechanical energy" fails because some energy of deformation is transformed into heat. Usually it is very difficult to predict precisely the dynamics of a seriously deformed rubber.

\subsection{Saint-Venant's principle}

Now we discuss the dynamics of rubber wiper blade. When a rubber wiper blade moves on the windshield of an automobile, some region $\Omega$ of the rubber wiper blade is only slightly deformed. See Figures 2.1 and 2.2. This phenomenon is usually considered as the effect of "Saint-Venant's principle". The Boussinesq version of "Saint-Venant's principle" can be expressed as follows.

"An equilibrated system of external forces applied to an elastic body, all of the points of application lying within a given sphere, produces deformations of negligible magnitude at distances from the sphere which are sufficiently large compared to its radius."

A mathematical formulation of this physical principle has been proved by Ernst 15 .

Let $D$ denote the corresponding 2-dimensional domain so that $\Omega$ is diffeomorphic to $[0, l] \times D$. See Figures 2.1 and 2.2 . On this slightly deformed region $\Omega$, the "hyperelasticity" of rubber may be assumed to be true [30]. Thus it is reasonable to predict the vibration of rubber wiper blade through mathematical analysis of the dynamics of this slightly deformed region $\Omega$.

\subsection{General physical principles on vibration}

General physical principles suggest that the dominant vibration frequencies of an elastic body are related to the "standing waves" on this elastic body [34]. This principle is 
generally adopted in the development of Quantum Physics 9]. Physicists considered "eigenstates" in Quantum Physics. The idea of "eigenstates" becomes the cornerstone of the Molecular Orbital Theory in Quantum Chemistry 21].

Therefore we will try to find the possible "eigenstates" in our analysis of the vibration of rubber wiper blade on convex windshield.

\section{Mathematical aspects of the vibration problem}

We will start with brief discussions on 3-dimensional elasticity mechanics with emphasis on Hyper-elasticity. Basic references for the elasticity mechanics are [8, 16].

Elasticity theory is based on the framework of continuum mechanics introduced by Cauchy. The key idea of elasticity theory is to relate "the infinitesimal variation of stress tensor" with "the infinitesimal deformation" of an elastic body.

Let $T(\mathbf{B})$ and $T^{*}(\mathbf{B})$ respectively denote the tangent bundle and the cotangent bundle of an elastic body $\mathbf{B}$. The Cauchy stress tensor $\mathbf{S}$ on an elastic body $\mathbf{B}$ is a section of

$$
T(\mathbf{B}) \otimes T^{*}(\mathbf{B})
$$

on $\mathbf{B}$. For a given unit tangent vector $\mathbf{v} \in T_{p}(\mathbf{B})$, we define

$$
\mathbf{v}^{+}=\left\{\mathbf{u} \in T_{p}(\mathbf{B}):\langle\mathbf{u}, \mathbf{v}\rangle=0\right\}
$$

to be the 2-dimensional subspace of $T_{p}(\mathbf{B})$ perpendicular to $\mathbf{v} . \mathbf{S}(\mathbf{v})$ indicates the pressure force acting on $\mathbf{v}^{+}$. We usually call "the component of $\mathbf{S}(\mathbf{v})$ along the $\mathbf{v}$-direction"

$$
\frac{\langle\mathbf{S}(\mathbf{v}), \mathbf{v}\rangle}{\|\mathbf{v}\|^{2}} \cdot \mathbf{v}
$$

"the normal stress component of $\mathbf{S}(\mathbf{v})$ ". We call "the component of $\mathbf{S}(\mathbf{v})$ perpendicular to the v-direction"

$$
-\frac{\langle\mathbf{S}(\mathbf{v}), \mathbf{v}\rangle}{\|\mathbf{v}\|^{2}} \cdot \mathbf{v}+\mathbf{S}(\mathbf{v})
$$

"the shear stress component of $\mathbf{S}(\mathbf{v})$ ".

\subsection{Differential geometry of elasticity mechanics}

In the following discussions, we will assume that $\mathbf{B}_{t}$ is a smooth family of 3-dimensional manifolds with boundary $\partial \mathbf{B}_{t}$ in $\mathbb{R}^{3}$ depending on the time variable $t . T\left(\mathbf{B}_{t}\right)$ and $T^{*}\left(\mathbf{B}_{t}\right)$ will respectively denote the tangent bundle and the cotangent bundle of $\mathbf{B}_{t}$. $\mathbf{S}$ is the $T\left(\mathbf{B}_{t}\right) \otimes T^{*}\left(\mathbf{B}_{t}\right)$-valued Cauchy stress tensor on $\mathbf{B}_{t}$. It should be noted that, according to the "conservation law of angular momentum", the Cauchy stress tensor $\mathbf{S}$ is symmetric 
when we identify $T^{*}\left(\mathbf{B}_{t}\right)$ with $T\left(\mathbf{B}_{t}\right)$ using the Euclidean metric on $\mathbb{R}^{3}$. See Appendix A of 16 .

In the following computation, $\mathbf{e}_{1}, \mathbf{e}_{2}, \mathbf{e}_{3}$ usually constitute an orthonormal framing field on $\mathbb{R}^{3}$. Since gravity is not important for the vibration of rubber wiper system, we will disregard the influence of gravity on the vibration of rubber wiper blade in this article.

According to the Hamilton principle, the motion of an elastic body must satisfy the variation formula

$$
-\delta \int_{t_{i}}^{t_{f}} U_{t} \cdot d t+\delta \int_{t_{i}}^{t_{f}} T_{t} \cdot d t+\int_{t_{i}}^{t_{f}}(\delta W)_{t} \cdot d t=0
$$

for any admissible smooth path of virtual deformation. Here $U_{t}$ is the potential corresponds to "the stored energy of elasticity" in the elastic body $\mathbf{B}_{t}$ at time $t$. See Appendix A of [16].

$T_{t}$ is the kinetic energy of $\mathbf{B}_{t}$. $(\delta W)_{t}$ corresponds to "the virtual work" done by the traction force $\tau_{t}$, along the boundary $\partial \mathbf{B}_{t}$ of $\mathbf{B}_{t}$, on the virtual deformation $(\delta \mathbf{x})_{t}$ at time $t$. We assume, for simplicity, that the density function $\rho_{t}$ of $\mathbf{B}_{t}$ does not depend substantially on $t$ so that

$$
\frac{\partial \rho_{t}}{\partial t} \approx 0
$$

It can be shown, using the Stokes Theorem and integration by parts, that the above variation formula is equivalent to

$$
\begin{aligned}
0= & -\delta \int_{t_{i}}^{t_{f}} U_{t} \cdot d t+\delta \int_{t_{i}}^{t_{f}} T_{t} \cdot d t+\int_{t_{i}}^{t_{f}}(\delta W)_{t} \cdot d t \\
= & \int_{t_{i}}^{t_{f}}\left(\oint_{\partial \mathbf{B}_{t}}\left\langle-\mathbf{S}\left(\mathbf{n}_{t}\right)+\tau_{t},(\delta \mathbf{x})_{t}\right\rangle\right) \cdot d t \\
& +\int_{t_{i}}^{t_{f}}\left(\int_{\mathbf{B}_{t}}\left\langle-\rho_{t} \cdot \frac{\partial}{\partial t} \frac{\partial \mathbf{u}}{\partial t}+\sum_{k=1}^{3}\left(\nabla_{\mathbf{e}_{k}} \mathbf{S}\right)\left(\mathbf{e}_{k}\right),(\delta \mathbf{x})_{t}\right\rangle\right) \cdot d t
\end{aligned}
$$

in which $(\delta \mathbf{x})_{t}$ is the admissible smooth path of virtual deformation depending on $t$. Here $\mathbf{n}_{t}$ is the outer normal vector field on the boundary $\partial \mathbf{B}_{t}$ of $\mathbf{B}_{t}$. In (3.1), $\mathbf{u}$ is the vectorvalued position function of $\mathbf{B}_{t} . \quad \nabla_{\mathbf{e}_{k}} \mathbf{S}$ is the covariant derivative of the Cauchy stress tensor $\mathbf{S}$ along the vector field $\mathbf{e}_{k}$. Thus the vector-valued Cauchy dynamic equation for the elastic body $\mathbf{B}_{t}$ is

$$
-\rho \cdot \frac{\partial^{2} \mathbf{u}}{\partial t^{2}}+\sum_{k=1}^{3}\left(\nabla_{\mathbf{e}_{k}} \mathbf{S}\right)\left(\mathbf{e}_{k}\right)=\mathbf{0} \quad \text { on } \mathbf{B}_{t}
$$

with

$$
\left.\mathbf{S}\left(\mathbf{n}_{t}\right)=\tau_{t} \text { (traction force given at time } t\right) \text { on } \partial \mathbf{B}_{t} \text {. }
$$


When a hyper-elastic body stays at an equilibrium state with traction acting on its boundary, we have the following system of (usually elliptic) partial differential equations

$$
\sum_{k=1}^{3}\left(\nabla_{\mathbf{e}_{k}} \mathbf{S}\right)\left(\mathbf{e}_{k}\right)=\mathbf{0} \quad \text { on } \mathbf{B}_{t}=\mathbf{B}_{0}
$$

with

$$
\mathbf{S}\left(\mathbf{n}_{t}\right)=\tau_{0} \quad \text { on } \partial \mathbf{B}_{t}=\partial \mathbf{B}_{0}
$$

independent of $t$.

Since the manifold $\mathbf{B}_{t}$ usually changes with $t$, it should be noted that the system 3.2 is not defined on a fixed region of $\mathbb{R}^{3}$. This is the fundamental reason why the system 3.2 is nonlinear in nature.

Now we explain how to transform the vector-valued Cauchy dynamic equation (3.2) into a vector-valued dynamic equation defined on the fixed region $\mathbf{B}_{0}$.

Let $\phi^{t}$ denote the diffeomorphism from $\mathbf{B}_{0}$ to $\mathbf{B}_{t}$ defined by the solution of the Cauchy dynamic equation (3.2). Let $\psi^{t}$ denote the corresponding inverse diffeomorphism from $\mathbf{B}_{t}$ to $\mathbf{B}_{0}$. We may define a family of stress tensors $\mathfrak{S}_{t}$ on $\mathbf{B}_{0}$, depending on $t$, by setting

$$
\mathfrak{S}_{t}=\psi_{*}^{t} \circ \mathbf{S} \circ \phi_{*}^{t}
$$

Here $\phi_{*}^{t}$ and $\psi_{*}^{t}$ are respectively the differential maps associated with the diffeomorphisms $\phi^{t}$ and $\psi^{t} . \mathfrak{S}_{t}$ is usually called the "second Piola-Kirchhoff stress tensor" associated with the Cauchy stress tensor $\mathbf{S}$ on $\mathbf{B}_{t}=\phi^{t}\left(\mathbf{B}_{0}\right)$. See Appendix A of $[16$.

Note that

$$
\mathbf{S}=\phi_{*}^{t} \circ \mathfrak{S}_{t} \circ \psi_{*}^{t}
$$

We may parameterize $\mathbf{B}_{t}=\phi^{t}\left(\mathbf{B}_{0}\right)$ by the points on $\mathbf{B}_{0}$. By doing so, we obtain the "first Piola-Kirchhoff stress tensor"

$$
\mathbf{S}_{\phi^{t}}
$$

associated with the Cauchy stress tensor $\mathbf{S}$ on $\mathbf{B}_{t}=\phi^{t}\left(\mathbf{B}_{0}\right)$.

We may express (3.2) as a system of equations defined on the fixed region $\mathbf{B}_{0}$ of $\mathbb{R}^{3}$ as follows. Let

$$
\mathbf{F}=\sum_{k=1}^{3}\left(\nabla_{\mathbf{e}_{k}} \mathbf{S}\right)\left(\mathbf{e}_{k}\right)=\sum_{k=1}^{3}\left[\nabla_{\mathbf{e}_{k}}\left(\phi_{*}^{t} \circ \mathfrak{S}_{t} \circ \psi_{*}^{t}\right)\right]\left(\mathbf{e}_{k}\right)
$$

denote the force field on $\mathbf{B}_{t}$ associated with the Cauchy stress tensor $\mathbf{S}$. Then we may express 3.2 as

$$
-\rho \cdot \frac{\partial^{2} \phi^{t}}{\partial t^{2}}+\mathbf{F}_{\phi^{t}}=\mathbf{0} \quad \text { on } \mathbf{B}_{0}
$$


in which $\mathbf{F}_{\phi^{t}}$ is the vector field on $\mathbf{B}_{t}=\phi^{t}\left(\mathbf{B}_{0}\right)$ parameterized by the points on $\mathbf{B}_{0}$. It can be observed readily that the system $(3.4)$ is naturally nonlinear. Note that the boundary condition 3.3 can be expressed as

$$
\mathfrak{S}_{t} \circ \psi_{*}^{t}\left(\mathbf{n}_{t}\right)=\psi_{*}^{t}\left(\tau_{t}\right) \text { on } \partial \mathbf{B}_{0}
$$

\subsection{Linearization of the nonlinear Cauchy dynamic equation}

To tackle the vibration problem of rubber wiper blade on convex windshield, we will consider the linearization of the nonlinear Cauchy dynamic equation on a slightly deformed region $\Omega$ of the rubber wiper blade. See Figures 2.1 and 2.2 .

Usually $\Omega$ is diffeomorphic to $[0, l] \times D$. Note that $\Omega$ is exactly $[0, l] \times D$ when the rubber wiper blade is at a static equilibrium state. According to the general principles of perturbation theory, it is reasonable to consider the following simplified problem: the linearization of the nonlinear Cauchy dynamic equation on $\Omega=[0, l] \times D$. We assume that the rubber material is homogeneous so that the following "Isotropy" condition (see Appendix D of [16]) is satisfied:

$$
\begin{aligned}
\left\langle(\delta \mathbf{S})\left(\mathbf{e}_{j}\right), \mathbf{e}_{k}\right\rangle & =\lambda \cdot\left(\frac{\partial v_{1}}{\partial x_{1}}+\frac{\partial v_{2}}{\partial x_{2}}+\frac{\partial v_{3}}{\partial x_{3}}\right) \cdot \delta_{j k}+2 \mu \cdot \epsilon_{j k} \\
& =\lambda \cdot(\operatorname{div} \mathbf{v}) \cdot \delta_{j k}+2 \mu \cdot \epsilon_{j k}
\end{aligned}
$$

with

$$
\epsilon_{j k}=\frac{1}{2}\left(\frac{\partial v_{k}}{\partial x_{j}}+\frac{\partial v_{j}}{\partial x_{k}}\right)
$$

Here $(\delta \mathbf{S})$ is "the infinitesimal variation of Cauchy stress tensor" corresponding to "the infinitesimal deformation" $\mathbf{v}(t, \mathbf{x})=\left(v_{1}(t, \mathbf{x}), v_{2}(t, \mathbf{x}), v_{3}(t, \mathbf{x})\right)$ on the hyper-elastic body $\mathbf{B}_{0}$. Here $\mathbf{x}=\left(x_{1}, x_{2}, x_{3}\right) \in \mathbf{B}_{0}$.

The linearization of (3.4) at a static equilibrium state can be expressed as the vectorvalued Lame equation:

$$
\rho \cdot \frac{\partial^{2} \mathbf{v}}{\partial t^{2}}=(\lambda+\mu) \cdot \nabla\left(\frac{\partial v_{1}}{\partial x_{1}}+\frac{\partial v_{2}}{\partial x_{2}}+\frac{\partial v_{3}}{\partial x_{3}}\right)+\mu \cdot\left(\frac{\partial^{2} \mathbf{v}}{\partial x_{1}^{2}}+\frac{\partial^{2} \mathbf{v}}{\partial x_{2}^{2}}+\frac{\partial^{2} \mathbf{v}}{\partial x_{3}^{2}}\right)
$$

in which $\rho$ is the (constant) density of the hyper-elastic body $\mathbf{B}_{0}$. Linearization of the boundary condition $(3.5)$ can be expressed as

$$
(\delta \mathbf{S})\left(\mathbf{n}_{0}\right)=(\delta \tau)
$$

Here the coefficients $\lambda$ and $\mu$ are Lame coefficients. See (1.3). 
3.3. Lame equation with specific physical boundary conditions

We assume, for simplicity, that $D$ is a 2-dimensional manifold with boundary on $\mathbb{R}^{2}$. Let

$$
\mathfrak{B}_{\Omega}=\left\{\left(x_{1}, x_{2}, x_{3}\right) \in \Omega: 0 \leq x_{1} \leq l \text { and }\left(x_{2}, x_{3}\right) \in \partial D\right\}
$$

Then the boundary $\partial \Omega$ of $\Omega=[0, l] \times D$ can be decomposed as follows:

$$
\partial \Omega=D_{0} \cup \mathfrak{B}_{\Omega} \cup D_{l}
$$

in which $D_{0}=\{0\} \times D$ and $D_{l}=\{l\} \times D$. Note that $\Omega=[0, l] \times D$ is not a 3 -dimensional manifold with boundary because $\partial \Omega$ contains the one-dimensional corner

$$
\operatorname{Corner}_{\Omega}=\left(\mathfrak{B}_{\Omega} \cap D_{0}\right) \cup\left(\mathfrak{B}_{\Omega} \cap D_{l}\right)
$$

When a rubber wiper blade moves on windshield, there is no stress or traction force acting along the outer normal vector field on $D_{0} \cup D_{l}$. This physical condition can be expressed as the boundary condition

$$
\left\langle(\delta \mathbf{S})\left(\mathbf{n}_{0}\right), \mathbf{n}_{0}\right\rangle=0 \quad \text { on } D_{0} \cup D_{l}
$$

at time $t$.

We assume that the rubber wiper blade moves on windshield at nearly a constant speed. To reduce the "wind resistance" on the windshield of a moving vehicle, the shape of windshield is generally strictly convex 7]. Thus we may assume that there is no frictional force acting on the ends $D_{0} \cup D_{l}$ of $\Omega=[0, l] \times D$. We may express this physical condition as the boundary condition

$$
\operatorname{div} \mathbf{v}=0 \quad \text { on } D_{0} \cup D_{l}
$$

at time $t$. See Section 4 for the explanation of 3.9 .

In the following analysis, we will discuss the solutions of the Lame equation

$$
\begin{aligned}
\frac{\partial^{2} \mathbf{v}}{\partial t^{2}} & =\frac{\lambda+\mu}{\rho} \cdot \nabla\left[\frac{\partial v_{1}}{\partial x_{1}}+\frac{\partial v_{2}}{\partial x_{2}}+\frac{\partial v_{3}}{\partial x_{3}}\right]+\frac{\mu}{\rho}\left[\frac{\partial^{2} \mathbf{v}}{\partial x_{1}^{2}}+\frac{\partial^{2} \mathbf{v}}{\partial x_{2}^{2}}+\frac{\partial^{2} \mathbf{v}}{\partial x_{3}^{2}}\right] \\
& =\frac{(\lambda+\mu)}{\rho} \cdot \nabla(\operatorname{div} \mathbf{v})+\frac{\mu}{\rho} \cdot \Delta \mathbf{v}
\end{aligned}
$$

on $\Omega=[0, l] \times D$, depending on $t$, satisfying the boundary conditions $(3.8)$ and $(3.9)$. Here we adopt the notation

$$
\Delta \equiv\left(\frac{\partial^{2}}{\partial x_{1}^{2}}+\frac{\partial^{2}}{\partial x_{2}^{2}}+\frac{\partial^{2}}{\partial x_{3}^{2}}\right)
$$

frequently used in PDE. Thus the operator $-\Delta$ is positive-definite. 
Proposition 3.1. Let I be an open interval for the time variable t. We have the following results.

(A) Assume that $\widetilde{w}(t, \mathbf{x})$ is a $C^{\infty}$ function defined on $I \times \Omega$ satisfying the wave equation

$$
\frac{\partial^{2} \widetilde{w}}{\partial t^{2}}-\frac{\lambda+2 \mu}{\rho} \cdot \Delta \widetilde{w}=0
$$

Then the deformation $\nabla \widetilde{w}(t, \mathbf{x})$ satisfies the Lame equation 3.10 :

$$
\frac{\partial^{2}(\nabla \widetilde{w})}{\partial t^{2}}=\frac{\lambda+\mu}{\rho} \cdot \nabla(\operatorname{div} \nabla \widetilde{w})+\frac{\mu}{\rho} \cdot \Delta(\nabla \widetilde{w}) .
$$

(B) Assume that $\mathbf{u}(t, \mathbf{x})$ is a $C^{\infty}$ vector-valued function defined on $I \times \Omega$ satisfying

$$
\operatorname{div} \mathbf{u}=0
$$

and the wave equation

$$
\frac{\partial^{2} \mathbf{u}}{\partial t^{2}}-\frac{\mu}{\rho} \cdot \Delta \mathbf{u}=0
$$

Then the deformation $\mathbf{u}(t, \mathbf{x})$ satisfies the Lame equation 3.10 :

$$
\frac{\partial^{2} \mathbf{u}}{\partial t^{2}}=\frac{\lambda+\mu}{\rho} \cdot \nabla(\operatorname{div} \mathbf{u})+\frac{\mu}{\rho} \cdot \Delta \mathbf{u} .
$$

Proof. This proposition can be checked readily using the equality $\Delta=\operatorname{div} \circ \nabla$.

We will show that a decomposition theorem (see Theorem 3.3 for the solutions $\mathbf{v}(t, \mathbf{x})$ of the Lame equation $(3.10)$ is possible. This means that we may express $\mathbf{v}(t, \mathbf{x})$ as the sum of $\nabla \widetilde{w}(t, \mathbf{x})$ and $\mathbf{u}(t, \mathbf{x})$ mentioned in Proposition 3.1. We start with the following preliminary result.

Lemma 3.2. Let $I$ be an open interval for the time variable $t$. Assume that $\mathbf{v}(t, \mathbf{x})$ is a $C^{\infty}$ vector-valued function defined on $I \times \Omega=I \times[0, l] \times D$ satisfying the Lame equation (3.10). Then there exists a unique function $w \in C^{\infty}\left(I, W_{0}^{1,2}(\Omega)\right)$ satisfying

$$
\Delta w=\operatorname{div} \mathbf{v}=\frac{\partial v_{1}}{\partial x_{1}}+\frac{\partial v_{2}}{\partial x_{2}}+\frac{\partial v_{3}}{\partial x_{3}}
$$

Here $W_{0}^{1,2}(\Omega)$ is the closure of $C_{0}^{1}(\Omega)$ in the Hilbert space $W^{1,2}(\Omega)$ consisting of weakly differentiable functions $f$ satisfying $\|f\|_{L^{2}(\Omega)}+\|\nabla f\|_{L^{2}(\Omega)}<+\infty$.

Proof. The unique existence of the function $w \in C^{\infty}\left(I, W_{0}^{1,2}(\Omega)\right)$ satisfying 3.10 follows from the general theory of elliptic partial differential equations. See Chapters 7 and 8 of 17 . 
Remark 3.1. According to the general theory of elliptic PDE, Chapter 8 of [17], the function $w$ of $(3.11)$ can be extended smoothly across the 2-dimensional portion of $\partial \Omega$ for each $t \in I$. However, the regularity of $w$ around the corner

$$
\operatorname{Corner}_{\Omega}=\left(\mathfrak{B}_{\Omega} \cap D_{0}\right) \cup\left(\mathfrak{B}_{\Omega} \cap D_{l}\right)
$$

of $\partial \Omega$ might be worse than usually expected. See $[12]$. Fortunately, this regularity problem is not very important for the applications of our theory.

Theorem 3.3 (Decomposition Theorem). Let I be an open interval for the time variable $t$. Assume that $\mathbf{v}(t, \mathbf{x})$ is a $C^{\infty}$ vector-valued function defined on $I \times \Omega$ satisfying the Lame equation (3.10). Then there exists a function $\widetilde{w}(t, \mathbf{x}) \in C^{\infty}\left(I, W_{0}^{1,2}(\Omega)\right)$ satisfying

$$
\Delta \widetilde{w}=\operatorname{div} \mathbf{v}
$$

and the wave equation

$$
\frac{\partial^{2} \widetilde{w}}{\partial t^{2}}-\frac{\lambda+2 \mu}{\rho} \cdot \Delta \widetilde{w}=0
$$

such that $\mathbf{v}(t, \mathbf{x})$ can be expressed as

$$
\mathbf{v}(t, \mathbf{x})=\mathbf{u}(t, \mathbf{x})+\nabla \widetilde{w}(t, \mathbf{x})
$$

in which $\mathbf{u}(t, \mathbf{x})$ is a vector-valued function on $I \times \Omega$ satisfying

$$
\operatorname{div} \mathbf{u}=0
$$

and the wave equation

$$
\frac{\partial^{2} \mathbf{u}}{\partial t^{2}}-\frac{\mu}{\rho} \cdot \Delta \mathbf{u}=0
$$

When the boundary condition $(3.9)$

$$
\operatorname{div} \mathbf{v}=0 \quad \text { on } I \times\left(D_{0} \cup D_{l}\right)
$$

is satisfied, we may require that $\widetilde{w}(t, \mathbf{x})$ satisfies the boundary condition

$$
\widetilde{w}=0 \quad \text { on } I \times\left(D_{0} \cup D_{l}\right) \text {. }
$$

Proof. Let $w \in C^{\infty}\left(I, W_{0}^{1,2}(\Omega)\right)$ denote the function, stated in Proposition 3.1, satisfying 3.11

$$
\Delta w=\operatorname{div} \mathbf{v}=\frac{\partial v_{1}}{\partial x_{1}}+\frac{\partial v_{2}}{\partial x_{2}}+\frac{\partial v_{3}}{\partial x_{3}}
$$


Let $\theta=\operatorname{div} \mathbf{v}$ so that $\Delta w=\operatorname{div} \mathbf{v}=\theta$. By taking the divergence of both sides of the Lame equation 3.10 , we obtain

$$
\frac{\partial^{2}(\operatorname{div} \mathbf{v})}{\partial t^{2}}=\operatorname{div} \circ \frac{\partial^{2} \mathbf{v}}{\partial t^{2}}=\frac{(\lambda+\mu)}{\rho} \cdot \operatorname{div} \circ \nabla(\operatorname{div} \mathbf{v})+\frac{\mu}{\rho} \cdot(\operatorname{div} \circ \Delta \mathbf{v})
$$

and so

$$
\frac{\partial^{2} \theta}{\partial t^{2}}=\frac{(\lambda+\mu)}{\rho} \cdot \operatorname{div} \circ \nabla \theta+\frac{\mu}{\rho} \cdot(\Delta \circ \operatorname{div} \mathbf{v})=\frac{(\lambda+\mu)}{\rho} \cdot \Delta \theta+\frac{\mu}{\rho} \cdot \Delta \theta .
$$

This means that $\theta=\operatorname{div} \mathbf{v}$ satisfies the wave equation

$$
\frac{\partial^{2} \theta}{\partial t^{2}}=\frac{(\lambda+2 \mu)}{\rho} \cdot \Delta \theta
$$

Since $\Delta w=\operatorname{div} \mathbf{v}=\theta$, it follows that

$$
\Delta\left(\frac{\partial^{2} w}{\partial t^{2}}-\frac{\lambda+2 \mu}{\rho} \cdot \Delta w\right)=\frac{\partial^{2} \Delta w}{\partial t^{2}}-\frac{\lambda+2 \mu}{\rho} \cdot \Delta \circ \Delta w=\frac{\partial^{2} \theta}{\partial t^{2}}-\frac{\lambda+2 \mu}{\rho} \cdot \Delta \theta=0
$$

and so the function

$$
h \equiv \frac{\partial^{2} w}{\partial t^{2}}-\frac{\lambda+2 \mu}{\rho} \cdot \Delta w=\frac{\partial^{2} w}{\partial t^{2}}-\frac{\lambda+2 \mu}{\rho} \cdot(\operatorname{div} \mathbf{v})
$$

is a $C^{\infty}$ family of space-harmonic functions depending on time $t \in I$.

Now we define a $C^{\infty}$ family $H(t, \mathbf{x})$ of space-harmonic functions depending on time $t \in I$ as follows:

$$
H(t, \mathbf{x}) \equiv \int_{0}^{t}\left(\int_{0}^{s} h(r, \mathbf{x}) \cdot d r\right) \cdot d s .
$$

Since $h$ is a $C^{\infty}$ family of space-harmonic functions depending on time $t \in I$, it is clear that

$$
\Delta H=0 \quad \text { and } \quad \frac{\partial^{2} H(t, \mathbf{x})}{\partial t^{2}}=h(t, \mathbf{x})
$$

We define $\widetilde{w}(t, \mathbf{x})=w(t, \mathbf{x})-H(t, \mathbf{x})$. Then we obtain (3.12):

$$
\Delta \widetilde{w}=\Delta w-\Delta H=(\operatorname{div} \mathbf{v})-0=\operatorname{div} \mathbf{v}
$$

It can be inferred from (3.19) and (3.17) that

$$
\frac{\partial^{2} H}{\partial t^{2}}-\frac{\lambda+2 \mu}{\rho} \cdot \Delta H=h-0=h \equiv \frac{\partial^{2} w}{\partial t^{2}}-\frac{\lambda+2 \mu}{\rho} \cdot \Delta w .
$$

It can be inferred readily from this equality that 3.13

$$
\frac{\partial^{2} \widetilde{w}}{\partial t^{2}}-\frac{\lambda+2 \mu}{\rho} \cdot \Delta \widetilde{w}=\frac{\partial^{2}(w-H)}{\partial t^{2}}-\frac{\lambda+2 \mu}{\rho} \cdot \Delta(w-H)=0
$$


is true.

When the boundary condition $(3.9)$

$$
\operatorname{div} \mathbf{v}=0 \quad \text { on } I \times\left(D_{0} \cup D_{l}\right)
$$

is satisfied, it can be observed readily from the definition 3.17) of $h(t, \mathbf{x})$ that

$$
h=\frac{\partial^{2} w}{\partial t^{2}}-\frac{\lambda+2 \mu}{\rho} \cdot(\operatorname{div} \mathbf{v})=0 \quad \text { on } D_{0} \cup D_{l}
$$

for each $t \in I$. Thus it follows from the definition 3.18 of $H(t, \mathbf{x})$ that

$$
H=0 \text { and so } \widetilde{w}=w-H=0 \text { on } I \times\left(D_{0} \cup D_{l}\right) .
$$

This proves 3.16 .

Now we define

$$
\mathbf{u}(t, \mathbf{x}) \equiv \mathbf{v}(t, \mathbf{x})-\nabla \widetilde{w}(t, \mathbf{x})
$$

so that $\mathbf{v}=\mathbf{u}+\nabla \widetilde{w}$. It is clear that $(3.14)$ is true:

$$
\operatorname{div} \mathbf{u} \equiv \operatorname{div} \mathbf{v}-\operatorname{div} \nabla \widetilde{w}=\operatorname{div} \mathbf{v}-\Delta \widetilde{w}=0
$$

Since $\mathbf{v}$ satisfies the Lame equation $(3.10)$, we have

$$
\frac{\partial^{2}(\mathbf{u}+\nabla \widetilde{w})}{\partial t^{2}}=\frac{\lambda+\mu}{\rho} \cdot \nabla(\operatorname{div} \mathbf{u}+\operatorname{div} \nabla \widetilde{w})+\frac{\mu}{\rho} \cdot \Delta(\mathbf{u}+\nabla \widetilde{w})
$$

in which $\mathbf{v}=\mathbf{u}+\nabla \widetilde{w}$. Since $\operatorname{div} \mathbf{u}=0$, we have

$$
\begin{aligned}
\frac{\partial^{2}(\mathbf{u}+\nabla \widetilde{w})}{\partial t^{2}} & =\frac{\lambda+\mu}{\rho} \cdot \nabla(0+\operatorname{div} \nabla \widetilde{w})+\frac{\mu}{\rho} \cdot \Delta(\mathbf{u}+\nabla \widetilde{w}) \\
& =\frac{\lambda+\mu}{\rho} \cdot \nabla(\Delta \widetilde{w})+\frac{\mu}{\rho} \cdot \Delta(\mathbf{u}+\nabla \widetilde{w})=\frac{\mu}{\rho} \cdot \Delta \mathbf{u}+\frac{\lambda+2 \mu}{\rho} \cdot \nabla(\Delta \widetilde{w}) .
\end{aligned}
$$

Since $\widetilde{w}$ satisfies the wave equation 3.13 , we infer from the above equality that

$$
\frac{\partial^{2} \mathbf{u}}{\partial t^{2}}-\frac{\mu}{\rho} \cdot \Delta \mathbf{u}=0
$$

This proves 3.15 .

Remark 3.2. The earliest version of the decomposition, using "grad" and "curl", of a vector field on $\mathbb{R}^{3}$ is due to $H$. von Helmholtz. Helmholtz Decomposition for vector fields on $\mathbb{R}^{3}$ was used by Maxwell in his theory of classical Electromagnetic Field. Helmholtz's work motivated the earlier development of Cohomology Theory and Hodge Theory. 
It should be noted that the condition $(3.16)$ of Theorem 3.3 is crucial for our theory. Usually the choice of boundary condition for PDE depends on the nature of the problem considered.

Recently, an existence theorem for the Lame equation, with nonlinear input force satisfying "null condition", was proved by Sideris and Klainerman under strict assumptions on the smallness of initial data. See [27,36]. The elasticity problem, considered by Klainerman and Sideris, is defined on $\mathbb{R}^{3}$ without specific boundary condition imposed.

Remark 3.3. In elasticity theory, there are "Saint-Venant compatibility conditions" related to the "overdeterminedness of stress distribution". Recently, it is known that these compatibility conditions are related to de Rham cohomology [1,2,13, 14].

We will call deformations, mentioned in Theorem 3.3, of the form

$$
\nabla \widetilde{w}(t, \mathbf{x}) \text { with } \widetilde{w} \text { satisfying } 3.12 \text { and } 3.13
$$

"*-exact deformations". We will call deformations, mentioned in Theorem 3.3 , of the form

$$
\mathbf{u}(t, \mathbf{x}) \text { with } \operatorname{div} \mathbf{u}=0 \text { 3.14 satisfying } 3.15
$$

"*-closed deformations".

Our next goal is to characterize the "*-exact deformations". We start with the following preliminary result.

Proposition 3.4. Let I be an open interval for the time variablet. Assume that $f\left(t, x_{2}, x_{3}\right)$ is a $C^{\infty}$ function defined on $I \times D$ satisfying the following wave equation

$$
\frac{\partial^{2} f}{\partial t^{2}}-\frac{\lambda+2 \mu}{\rho} \cdot\left(\frac{-n^{2} \cdot \pi^{2}}{l^{2}} \cdot f+\frac{\partial^{2} f}{\partial x_{2}^{2}}+\frac{\partial^{2} f}{\partial x_{3}^{2}}\right)=0
$$

in which $n$ is a positive integer. Then the function $\widetilde{w}(t, \mathbf{x})$ defined by

$$
\widetilde{w}\left(t, x_{1}, x_{2}, x_{3}\right) \equiv f\left(t, x_{2}, x_{3}\right) \cdot \sqrt{\frac{2}{l}} \cdot \sin \left(\frac{n \cdot \pi \cdot x_{1}}{l}\right)
$$

is a solution of the wave equation 3.13

$$
\frac{\partial^{2} \widetilde{w}}{\partial t^{2}}-\frac{\lambda+2 \mu}{\rho} \cdot \Delta \widetilde{w}=0
$$

satisfying the boundary condition 3.16

$$
\widetilde{w}=0 \quad \text { on } I \times\left(D_{0} \cup D_{l}\right) \text {. }
$$

Let $\mathbf{v}(t, \mathbf{x})=\nabla \widetilde{w}(t, \mathbf{x})$. Then the deformation $\mathbf{v}(t, \mathbf{x})=\nabla \widetilde{w}(t, \mathbf{x})$ is a solution of the Lame equation (3.10) satisfying the boundary conditions (3.8) and (3.9) at time $t \in I$. 
Proof. It can be inferred readily from $(3.20)$ that $\widetilde{w}\left(t, x_{1}, x_{2}, x_{3}\right)$ satisfies the wave equation 3.13 . Since

$$
\sin \left(\frac{n \cdot \pi \cdot 0}{l}\right)=\sin \left(\frac{n \cdot \pi \cdot l}{l}\right)
$$

it is clear that $\widetilde{w}\left(t, x_{1}, x_{2}, x_{3}\right)$ satisfies the boundary condition (3.16). Since

$$
\operatorname{div} \mathbf{v}=\Delta \widetilde{w}=\left(\frac{-n^{2} \cdot \pi^{2}}{l^{2}} \cdot f+\frac{\partial^{2} f}{\partial x_{2}^{2}}+\frac{\partial^{2} f}{\partial x_{3}^{2}}\right) \cdot \sin \left(\frac{n \cdot \pi \cdot x_{1}}{l}\right),
$$

it is clear that the boundary condition (3.9) is satisfied by $\mathbf{v}(t, \mathbf{x})=\nabla \widetilde{w}(t, \mathbf{x})$ because of 3.22 .

Now we consider the boundary condition $(3.8)$. Since the boundary condition $(3.9)$ is satisfied, it follows from $(3.6)$ and 3.7 that

$$
\begin{aligned}
\left\langle(\delta \mathbf{S})\left(\mathbf{e}_{j}\right), \mathbf{e}_{k}\right\rangle & =\lambda \cdot(\operatorname{div} \mathbf{v}) \cdot \delta_{j k}+2 \mu \cdot \epsilon_{j k} \\
& =0+\mu \cdot\left(\frac{\partial v_{k}}{\partial x_{j}}+\frac{\partial v_{j}}{\partial x_{k}}\right)=2 \mu \cdot \frac{\partial^{2} \widetilde{w}}{\partial x_{j} \partial x_{k}}
\end{aligned}
$$

on $I \times\left(D_{0} \cup D_{l}\right)$. Since $\mathbf{n}_{0}=\mp \mathbf{e}_{1}$ on $D_{0} \cup D_{l}$, we have

$$
\left\langle(\delta \mathbf{S})\left(\mathbf{n}_{0}\right), \mathbf{n}_{0}\right\rangle=2 \mu \cdot \frac{\partial^{2} \widetilde{w}}{\partial x_{1} \partial x_{1}}=0
$$

because of 3.22 .

Since the wave equation $(3.13)$ and the pair of boundary conditions $(3.8)$ and 3.9 are all linear, it is clear that any convergent linear combination of functions of the form (3.21) will give rise to a solution of the wave equation (3.13) satisfying the pair of boundary conditions (3.8) and (3.9). Our next main theorem shows that these are exactly the only solutions for the wave equation (3.13) satisfying the pair of boundary conditions $(3.8)$ and (3.9.

Theorem 3.5 (characterization of $*$-exact deformations). Let $I$ be an open interval for the time variable $t$. Assume that $\widetilde{w}(t, \mathbf{x})$ is a $C^{\infty}$ solution of the wave equation (3.13)

$$
\frac{\partial^{2} \widetilde{w}}{\partial t^{2}}-\frac{\lambda+2 \mu}{\rho} \cdot \Delta \widetilde{w}=0
$$

on $I \times \Omega$ satisfying the boundary condition 3.16

$$
\widetilde{w}=0 \quad \text { on } I \times\left(D_{0} \cup D_{l}\right)
$$

mentioned in Theorem 3.3. Assume that the boundary condition (3.9)

$$
\operatorname{div} \nabla \widetilde{w}=0 \quad \text { on } D_{0} \cup D_{l}
$$


and the boundary condition (3.8)

$$
\left\langle(\delta \mathbf{S})\left(\mathbf{n}_{0}\right), \mathbf{n}_{0}\right\rangle=0 \quad \text { on } D_{0} \cup D_{l}
$$

are satisfied by the deformation $\nabla \widetilde{w}(t, \mathbf{x})$ at any time $t \in I$. Then the function $\widetilde{w}(t, \mathbf{x})$ can be expressed as

$$
\widetilde{w}(t, \mathbf{x})=\sum_{n=1}^{\infty} \Gamma_{n}\left(t, x_{2}, x_{3}\right) \cdot \sqrt{\frac{2}{l}} \cdot \sin \left(\frac{n \cdot \pi \cdot x_{1}}{l}\right)
$$

in which the function

$$
\Gamma_{n}\left(t, x_{2}, x_{3}\right)=\int_{0}^{l} \widetilde{w}\left(t, s, x_{2}, x_{3}\right) \cdot \sqrt{\frac{2}{l}} \cdot \sin \left(\frac{n \cdot \pi \cdot s}{l}\right) \cdot d s
$$

satisfies the wave equation (3.20)

$$
\frac{\partial^{2} \Gamma_{n}}{\partial t^{2}}-\frac{\lambda+2 \mu}{\rho} \cdot\left(\frac{-n^{2} \cdot \pi^{2}}{l^{2}} \cdot \Gamma_{n}+\frac{\partial^{2} \Gamma_{n}}{\partial x_{2}^{2}}+\frac{\partial^{2} \Gamma_{n}}{\partial x_{3}^{2}}\right)=0 .
$$

Proof. Since $\operatorname{div} \nabla \widetilde{w}=0$ on $D_{0} \cup D_{l}$, it follows from (3.6) and (3.7) that

$$
\left\langle(\delta \mathbf{S})\left(\mathbf{e}_{j}\right), \mathbf{e}_{k}\right\rangle=\lambda \cdot(\operatorname{div} \mathbf{v}) \cdot \delta_{j k}+2 \mu \cdot \epsilon_{j k}=0+2 \mu \cdot \frac{\partial^{2} \widetilde{w}}{\partial x_{j} \partial x_{k}}
$$

on $D_{0} \cup D_{l}$ at any time $t \in I$. Since $\mathbf{n}_{0}=\mp \mathbf{e}_{1}$ on $D_{0} \cup D_{l}$, the condition $\left\langle(\delta \mathbf{S})\left(\mathbf{n}_{0}\right), \mathbf{n}_{0}\right\rangle=0$ on $D_{0} \cup D_{l}$ can be expressed as

$$
0=\left\langle(\delta \mathbf{S})\left(\mathbf{n}_{0}\right), \mathbf{n}_{0}\right\rangle=2 \mu \cdot \frac{\partial^{2} \widetilde{w}}{\partial x_{1}^{2}}=0 \quad \text { on } D_{0} \cup D_{l} .
$$

Thus we have

$$
\frac{\partial^{2} \widetilde{w}}{\partial x_{1}^{2}}\left(t, 0, x_{2}, x_{3}\right)=0=\frac{\partial^{2} \widetilde{w}}{\partial x_{1}^{2}}\left(t, l, x_{2}, x_{3}\right)
$$

at any time $t \in I$. It follows from the spectral theory that we have

$$
\frac{\partial^{2} \widetilde{w}}{\partial x_{1}^{2}}(t, \mathbf{x})=\sum_{n=1}^{\infty} \gamma_{n}\left(t, x_{2}, x_{3}\right) \cdot \sqrt{\frac{2}{l}} \cdot \sin \left(\frac{n \cdot \pi \cdot x_{1}}{l}\right)
$$

in which

$$
\gamma_{n}\left(t, x_{2}, x_{3}\right)=\int_{0}^{l} \frac{\partial^{2} \widetilde{w}}{\partial x_{1}^{2}}\left(t, s, x_{2}, x_{3}\right) \cdot \sqrt{\frac{2}{l}} \cdot \sin \left(\frac{n \cdot \pi \cdot s}{l}\right) \cdot d s
$$

is the $L^{2}$ projection of $\frac{\partial^{2} \widetilde{w}}{\partial x_{1}^{2}}$ on the eigenfunction

$$
\sqrt{\frac{2}{l}} \cdot \sin \left(\frac{n \cdot \pi \cdot s}{l}\right)
$$


for the Dirichlet problem of one-dimensional Laplace operator on $[0, l]$. It should be noted that the convergence of the Fourier series (3.24) is uniform. See Corollary 10.4 of [35].

Since $\widetilde{w}=0$ on $D_{0} \cup D_{l}$ at any time $t \in I$, we have

$$
\widetilde{w}(t, \mathbf{x})=\sum_{n=1}^{\infty} \Gamma_{n}\left(t, x_{2}, x_{3}\right) \cdot \sqrt{\frac{2}{l}} \cdot \sin \left(\frac{n \cdot \pi \cdot x_{1}}{l}\right)
$$

in which

$$
\Gamma_{n}\left(t, x_{2}, x_{3}\right)=\int_{0}^{l} \widetilde{w}\left(t, s, x_{2}, x_{3}\right) \cdot \sqrt{\frac{2}{l}} \cdot \sin \left(\frac{n \cdot \pi \cdot s}{l}\right) \cdot d s .
$$

Note, by using integration by parts twice, that

$$
\gamma_{n}=-\frac{n^{2} \pi^{2}}{l^{2}} \cdot \int_{0}^{l} \widetilde{w}\left(t, s, x_{2}, x_{3}\right) \cdot \sqrt{\frac{2}{l}} \cdot \sin \left(\frac{n \pi \cdot s}{l}\right) \cdot d s=-\frac{n^{2} \pi^{2}}{l^{2}} \cdot \Gamma_{n} .
$$

Since $\widetilde{w}=0$ on $D_{0} \cup D_{l}$ at any time $t \in I$, we have

$$
\frac{\partial^{2} \widetilde{w}}{\partial x_{2}^{2}}=\frac{\partial^{2} \widetilde{w}}{\partial x_{3}^{2}}=0=\frac{\partial^{2} \widetilde{w}}{\partial t^{2}} \quad \text { on } D_{0} \cup D_{l}
$$

at any time $t \in I$. Thus we have

$$
\begin{aligned}
\frac{\partial^{2} \widetilde{w}}{\partial x_{2}^{2}}(t, \mathbf{x}) & =\sum_{n=1}^{\infty} \sqrt{\frac{2}{l}} \sin \left(\frac{n \pi x_{1}}{l}\right) \cdot \int_{0}^{l} \frac{\partial^{2} \widetilde{w}}{\partial x_{2}^{2}}\left(t, s, x_{2}, x_{3}\right) \cdot \sqrt{\frac{2}{l}} \sin \left(\frac{n \pi s}{l}\right) \cdot d s \\
& =\sum_{n=1}^{\infty} \sqrt{\frac{2}{l}} \cdot \sin \left(\frac{n \cdot \pi \cdot x_{1}}{l}\right) \cdot \frac{\partial^{2} \Gamma_{n}}{\partial x_{2}^{2}}\left(t, x_{2}, x_{3}\right)
\end{aligned}
$$

and

$$
\begin{aligned}
\frac{\partial^{2} \widetilde{w}}{\partial x_{3}^{2}}(t, \mathbf{x}) & =\sum_{n=1}^{\infty} \sqrt{\frac{2}{l}} \sin \left(\frac{n \pi x_{1}}{l}\right) \cdot \int_{0}^{l} \frac{\partial^{2} \widetilde{w}}{\partial x_{3}^{2}}\left(t, s, x_{2}, x_{3}\right) \cdot \sqrt{\frac{2}{l}} \sin \left(\frac{n \pi s}{l}\right) \cdot d s \\
& =\sum_{n=1}^{\infty} \sqrt{\frac{2}{l}} \cdot \sin \left(\frac{n \cdot \pi \cdot x_{1}}{l}\right) \cdot \frac{\partial^{2} \Gamma_{n}}{\partial x_{3}^{2}}\left(t, x_{2}, x_{3}\right) .
\end{aligned}
$$

Similarly we have

$$
\begin{aligned}
\frac{\partial^{2} \widetilde{w}}{\partial t^{2}}(t, \mathbf{x}) & =\sum_{n=1}^{\infty} \sqrt{\frac{2}{l}} \sin \left(\frac{n \pi x_{1}}{l}\right) \cdot \int_{0}^{l} \frac{\partial^{2} \widetilde{w}}{\partial t^{2}}\left(t, s, x_{2}, x_{3}\right) \cdot \sqrt{\frac{2}{l}} \sin \left(\frac{n \pi s}{l}\right) \cdot d s \\
& =\sum_{n=1}^{\infty} \sqrt{\frac{2}{l}} \cdot \sin \left(\frac{n \cdot \pi \cdot x_{1}}{l}\right) \cdot \frac{\partial^{2} \Gamma_{n}}{\partial t^{2}}\left(t, x_{2}, x_{3}\right) .
\end{aligned}
$$

It can be inferred readily from $3.25,(3.26),(3.27)$ and 3.28 that the wave equation (3.13) can be expressed as

$$
\begin{aligned}
0 & =\frac{\partial^{2} \widetilde{w}}{\partial t^{2}}-\frac{\lambda+2 \mu}{\rho} \cdot \Delta \widetilde{w} \\
& =\sum_{n=1}^{\infty} \sqrt{\frac{2}{l}} \sin \left(\frac{n \pi x_{1}}{l}\right) \cdot\left[\frac{\partial^{2} \Gamma_{n}}{\partial t^{2}}-\frac{\lambda+2 \mu}{\rho}\left(\frac{-n^{2} \pi^{2} \Gamma_{n}}{l^{2}}+\frac{\partial^{2} \Gamma_{n}}{\partial x_{2}^{2}}+\frac{\partial^{2} \Gamma_{n}}{\partial x_{3}^{2}}\right)\right]
\end{aligned}
$$


In particular, the function $\Gamma_{n}\left(t, x_{2}, x_{3}\right)$ must satisfy the wave equation 3.20

$$
\frac{\partial^{2} \Gamma_{n}}{\partial t^{2}}-\frac{\lambda+2 \mu}{\rho} \cdot\left(\frac{-n^{2} \cdot \pi^{2} \cdot \Gamma_{n}}{l^{2}}+\frac{\partial^{2} \Gamma_{n}}{\partial x_{2}^{2}}+\frac{\partial^{2} \Gamma_{n}}{\partial x_{3}^{2}}\right)=0 .
$$

Remark 3.4. Theorem 3.5 shows us that the solutions of Class I may be classified by the "shear index" $n$. We say that a solution of Class I has shear index $n$ if and only if this solution can be expressed as

$$
\Gamma_{n}\left(t, x_{2}, x_{3}\right) \cdot \sqrt{\frac{2}{l}} \cdot \sin \left(\frac{n \cdot \pi \cdot x_{1}}{l}\right)
$$

with the function $\Gamma_{n}\left(t, x_{2}, x_{3}\right)$ satisfying the wave equation 3.20$)$.

Now we discuss "*-closed deformations". Unlike "*-exact deformations", simple and useful characterization of "*-closed deformations" does not seem possible. In fact, a cohomological characterization of "*-closed deformations" is possible but unlikely to be useful, because we are most concerned about the "vibration frequencies" of such deformations. Our next few propositions will be useful to help us to understand that " $*$-closed deformations" have diverse vibration frequencies.

Proposition 3.6. Let $I$ be an open interval for the time variable $t$. Assume that $\mathbf{u}(t, \mathbf{x})=$ $\left(u_{1}(t, \mathbf{x}), u_{2}(t, \mathbf{x}), u_{3}(t, \mathbf{x})\right)$ is a $C^{\infty}$ vector-valued function defined on $I \times \Omega$ with (3.14)

$$
\operatorname{div} \mathbf{u}=0
$$

satisfying the wave equation 3.15

$$
\frac{\partial^{2} \mathbf{u}}{\partial t^{2}}-\frac{\mu}{\rho} \cdot \Delta \mathbf{u}=0
$$

Then the boundary condition 3.8

$$
\left\langle(\delta \mathbf{S})\left(\mathbf{n}_{0}\right), \mathbf{n}_{0}\right\rangle=0 \quad \text { on } D_{0} \cup D_{l}
$$

for the deformation $\mathbf{u}(t, \mathbf{x})$ is satisfied if and only if

$$
\frac{\partial u_{1}}{\partial x_{1}}\left(t, 0, x_{2}, x_{3}\right)=0=\frac{\partial u_{1}}{\partial x_{1}}\left(t, l, x_{2}, x_{3}\right) .
$$

When the boundary condition (3.8) is satisfied by $\mathbf{u}(t, \mathbf{x})$, the function $u_{1}(t, \mathbf{x})$ can be expressed as a uniformly convergent series

$$
u_{1}\left(t, x_{1}, x_{2}, x_{3}\right)=\widetilde{c}_{0}\left(t, x_{2}, x_{3}\right)+\sum_{n=1}^{\infty} \widetilde{c}_{n}\left(t, x_{2}, x_{3}\right) \cdot \cos \left(\frac{n \cdot \pi \cdot x_{1}}{l}\right) .
$$


Proof. Since div $\mathbf{u}=0$, it follows from (3.6) and (3.7) that

$$
\left\langle(\delta \mathbf{S})\left(\mathbf{e}_{j}\right), \mathbf{e}_{k}\right\rangle=\lambda \cdot(\operatorname{div} \mathbf{u}) \cdot \delta_{j k}+2 \mu \cdot \epsilon_{j k}=0+\mu \cdot\left(\frac{\partial u_{k}}{\partial x_{j}}+\frac{\partial u_{j}}{\partial x_{k}}\right)
$$

on $D_{0} \cup D_{l}$ at any time $t \in I$. Since $\mathbf{n}_{0}=\mp \mathbf{e}_{1}$ on $D_{0} \cup D_{l}$, the boundary condition (3.8) $\left\langle(\delta \mathbf{S})\left(\mathbf{n}_{0}\right), \mathbf{n}_{0}\right\rangle=0$ on $D_{0} \cup D_{l}$ can be expressed as

$$
0=\left\langle(\delta \mathbf{S})\left(\mathbf{n}_{0}\right), \mathbf{n}_{0}\right\rangle=2 \mu \cdot \frac{\partial u_{1}}{\partial x_{1}} \quad \text { on } D_{0} \cup D_{l}
$$

at any time $t \in I$. Thus the boundary condition $(3.8)$ for the deformation $\mathbf{u}(t, \mathbf{x})$ is satisfied if and only if 3.29

$$
\frac{\partial u_{1}}{\partial x_{1}}\left(t, 0, x_{2}, x_{3}\right)=0=\frac{\partial u_{1}}{\partial x_{1}}\left(t, l, x_{2}, x_{3}\right)
$$

is true. When the boundary condition (3.8) for $\mathbf{u}(t, \mathbf{x})$ is satisfied, it follows from the spectral theory that we have

$$
\frac{\partial u_{1}}{\partial x_{1}}(t, \mathbf{x})=\sum_{n=1}^{\infty} c_{n}\left(t, x_{2}, x_{3}\right) \cdot \sqrt{\frac{2}{l}} \cdot \sin \left(\frac{n \cdot \pi \cdot x_{1}}{l}\right)
$$

in which

$$
c_{n}\left(t, x_{2}, x_{3}\right)=\int_{0}^{l} \frac{\partial u_{1}}{\partial x_{1}}\left(t, s, x_{2}, x_{3}\right) \cdot \sqrt{\frac{2}{l}} \cdot \sin \left(\frac{n \cdot \pi \cdot s}{l}\right) \cdot d s .
$$

Note that the Hölder continuity of $\frac{\partial u_{1}}{\partial x_{1}}(t, \mathbf{x})$ in the variable $x_{1}$ suffices to ensure that the series 3.31) converges uniformly. See Corollary 10.4 of 35]. Thus we have

$$
\begin{aligned}
u_{1}\left(t, x_{1}, x_{2}, x_{3}\right) & =u_{1}\left(t, 0, x_{2}, x_{3}\right)+\int_{0}^{x_{1}} \frac{\partial u_{1}}{\partial x_{1}}\left(t, s, x_{2}, x_{3}\right) \cdot d s \\
& =u_{1}\left(t, 0, x_{2}, x_{3}\right)+\sum_{n=1}^{\infty} c_{n}\left(t, x_{2}, x_{3}\right) \cdot \sqrt{\frac{2}{l}} \cdot \int_{0}^{x_{1}} \sin \left(\frac{n \cdot \pi \cdot s}{l}\right) \cdot d s \\
& =\widetilde{c}_{0}\left(t, x_{2}, x_{3}\right)+\sum_{n=1}^{\infty} \widetilde{c}_{n}\left(t, x_{2}, x_{3}\right) \cdot \cos \left(\frac{n \cdot \pi \cdot x_{1}}{l}\right)
\end{aligned}
$$

in which

$$
\widetilde{c}_{0}\left(t, x_{2}, x_{3}\right)=u_{1}\left(t, 0, x_{2}, x_{3}\right)+\sum_{n=1}^{\infty} c_{n}\left(t, x_{2}, x_{3}\right) \cdot \sqrt{\frac{2}{l}} \cdot \frac{l}{n \cdot \pi}
$$

and

$$
\widetilde{c}_{n}\left(t, x_{2}, x_{3}\right)=-\sqrt{\frac{2}{l}} \cdot \frac{l}{n \cdot \pi} \cdot c_{n}\left(t, x_{2}, x_{3}\right)
$$

for each positive integer $n$. This completes the proof of 3.30. 
Remark 3.5. In Theorem 3.5, we know that the functions $\Gamma_{n}\left(t, x_{2}, x_{3}\right)$ must satisfy specific wave equations. Unlike Theorem 3.5, Proposition 3.6 does not tell us what the functions $\widetilde{c}_{n}\left(t, x_{2}, x_{3}\right)$ should be.

Our next two propositions will show us how elementary "*-closed deformations" can be constructed. These elementary "*-closed deformations", constructed in Propositions 3.7 and 3.8, may help us understand the vibration frequencies of rubber wiper blade on convex windshield.

Proposition 3.7. Let $I$ be an open interval for the time variable $t$. Assume that $f\left(t, x_{2}, x_{3}\right)$ is a $C^{\infty}$ function defined on $I \times D$ satisfying the following wave equation

$$
\frac{\partial^{2} f}{\partial t^{2}}-\frac{\mu}{\rho} \cdot\left(\frac{-n^{2} \cdot \pi^{2}}{l^{2}} \cdot f+\frac{\partial^{2} f}{\partial x_{2}^{2}}+\frac{\partial^{2} f}{\partial x_{3}^{2}}\right)=0
$$

in which $n$ is a positive integer. Let $\mathbf{u}(t, \mathbf{x})=\left(u_{1}(t, \mathbf{x}), u_{2}(t, \mathbf{x}), u_{3}(t, \mathbf{x})\right)$ denote the $C^{\infty}$ vector-valued function defined on $I \times \Omega$ as follows:

$$
u_{1}(t, \mathbf{x})=\frac{l}{n \cdot \pi} \cdot \cos \left(\frac{n \cdot \pi \cdot x_{1}}{l}\right) \cdot\left(\frac{\partial^{2} f}{\partial x_{2}^{2}}+\frac{\partial^{2} f}{\partial x_{3}^{2}}\right)
$$

with

$$
u_{2}(t, \mathbf{x})=\sin \left(\frac{n \cdot \pi \cdot x_{1}}{l}\right) \cdot \frac{\partial f}{\partial x_{2}} \quad \text { and } \quad u_{3}(t, \mathbf{x})=\sin \left(\frac{n \cdot \pi \cdot x_{1}}{l}\right) \cdot \frac{\partial f}{\partial x_{3}} .
$$

Then this deformation $\mathbf{u}(t, \mathbf{x})$ satisfies (3.14

$$
\operatorname{div} \mathbf{u}=0
$$

and the wave equation 3.15

$$
\frac{\partial^{2} \mathbf{u}}{\partial t^{2}}-\frac{\mu}{\rho} \cdot \Delta \mathbf{u}=0
$$

Moreover, it satisfies the boundary condition (3.8)

$$
\left\langle(\delta \mathbf{S})\left(\mathbf{n}_{0}\right), \mathbf{n}_{0}\right\rangle=0 \quad \text { on } D_{0} \cup D_{l}
$$

because

$$
\frac{\partial u_{1}}{\partial x_{1}}\left(t, 0, x_{2}, x_{3}\right)=0=\frac{\partial u_{1}}{\partial x_{1}}\left(t, l, x_{2}, x_{3}\right) .
$$

Proof. This proposition can be checked directly.

Proposition 3.8. Let $I$ be an open interval for the time variable $t$. Assume that $g(t, \mathbf{x})$ is a $C^{\infty}$ function on $I \times \Omega$ satisfying the wave equation

$$
\frac{\partial^{2} g}{\partial t^{2}}-\frac{\mu}{\rho} \cdot \Delta g=0
$$


Then the deformation $\mathbf{u}(t, \mathbf{x})=\left(u_{1}(t, \mathbf{x}), u_{2}(t, \mathbf{x}), u_{3}(t, \mathbf{x})\right)$ defined by

$$
\mathbf{u}(t, \mathbf{x})=\left(0,-\frac{\partial g}{\partial x_{3}}(t, \mathbf{x}), \frac{\partial g}{\partial x_{2}}(t, \mathbf{x})\right)
$$

satisfies 3.14

$$
\operatorname{div} \mathbf{u}=0
$$

and the wave equation 3.15

$$
\frac{\partial^{2} \mathbf{u}}{\partial t^{2}}-\frac{\mu}{\rho} \cdot \Delta \mathbf{u}=0
$$

Moreover, it satisfies the boundary condition (3.8)

$$
\left\langle(\delta \mathbf{S})\left(\mathbf{n}_{0}\right), \mathbf{n}_{0}\right\rangle=0 \quad \text { on } D_{0} \cup D_{l}
$$

because $u_{1}(t, \mathbf{x})=0$.

Proof. This proposition can be checked directly.

\section{Physical explanation}

The pressure on the rubber wiper blade comes from the Wiper-Arm. This force only acts on some sites of the rubber wiper blade. To reduce the "wind resistance" on the windshield of a moving vehicle, the shape of windshield is generally strictly convex [7]. Since the windshield is strictly convex with (usually small) positive sectional curvature, the ends of rubber wiper blade usually do not touch the convex windshield. See Figure 4.1.

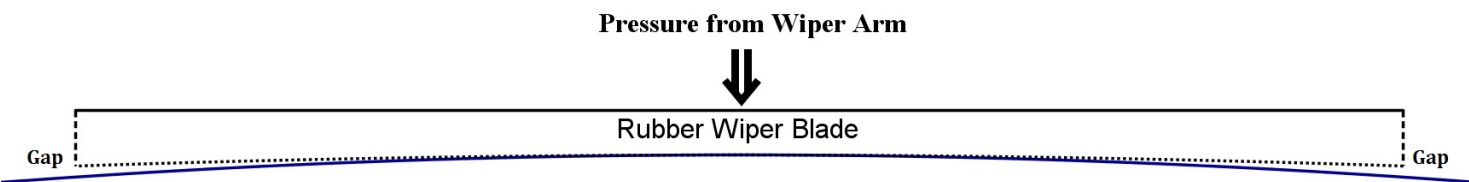

Convex Windshield with Positive Sectional Curvature

Figure 4.1: Rubber wiper blade on strictly convex windshield.

Thus we may deduce from the Saint-Venant principle, mentioned in Section 2, that "the infinitesimal variation of volume", associated with the infinitesimal deformation $\mathbf{v}$, around the ends of rubber wiper blade is zero (3.9):

$$
\operatorname{div} \mathbf{v}=0 \quad \text { on } D_{0} \cup D_{l}
$$


More explanation on the mechanics of rubber wiper blade on convex windshield can be found in 11$]$.

Now we explain why Theorem 3.5 (characterization of $*$-exact deformations) leads to the prediction that most of the vibration frequencies of Class I (*-exact deformations) should locate around (1.1):

$$
\sqrt{\frac{\lambda+2 \mu}{\rho}} \cdot \frac{n}{2 l} \mathrm{~Hz} \quad(\text { Class I })
$$

where $n$ is a positive integer. Theorem 3.5 tells us that each general solution $\widetilde{w}(t, \mathbf{x})$ of Class I can be expressed as 3.23 :

$$
\widetilde{w}(t, \mathbf{x})=\sum_{n=1}^{\infty} \Gamma_{n}\left(t, x_{2}, x_{3}\right) \cdot \sqrt{\frac{2}{l}} \cdot \sin \left(\frac{n \cdot \pi \cdot x_{1}}{l}\right)
$$

in which the function $\Gamma_{n}\left(t, x_{2}, x_{3}\right)$ satisfies the wave equation 3.20

$$
\frac{\partial^{2} \Gamma_{n}}{\partial t^{2}}-\frac{\lambda+2 \mu}{\rho} \cdot\left(\frac{-n^{2} \cdot \pi^{2}}{l^{2}} \cdot \Gamma_{n}+\frac{\partial^{2} \Gamma_{n}}{\partial x_{2}^{2}}+\frac{\partial^{2} \Gamma_{n}}{\partial x_{3}^{2}}\right)=0
$$

When a rubber wiper blade moves on the windshield of an automobile, the kinetic frictional force, acting on the lower part of rubber wiper blade, causes the rubber wiper blade to vibrate. From the physical point of view, it is reasonable to consider eigenfunctions $\Gamma_{n}\left(t, x_{2}, x_{3}\right)=e^{i \cdot k \cdot t} \cdot \gamma_{n}\left(x_{2}, x_{3}\right)$ satisfying

$$
\frac{\partial^{2} \Gamma_{n}}{\partial x_{2}^{2}}+\frac{\partial^{2} \Gamma_{n}}{\partial x_{3}^{2}}=-\epsilon_{n} \cdot \Gamma_{n} \quad \text { or } \quad \frac{\partial^{2} \gamma_{n}}{\partial x_{2}^{2}}+\frac{\partial^{2} \gamma_{n}}{\partial x_{3}^{2}}=-\epsilon_{n} \cdot \gamma_{n}
$$

Substituting (4.1) into the wave equation 3.20, we infer that

$$
-k^{2}-\frac{\lambda+2 \mu}{\rho} \cdot\left[\frac{-n^{2} \cdot \pi^{2}}{l^{2}}-\epsilon_{n}\right]=0 \quad \text { or } \quad k= \pm \sqrt{\frac{\lambda+2 \mu}{\rho}} \cdot \sqrt{\frac{n^{2} \cdot \pi^{2}}{l^{2}}+\epsilon_{n}} .
$$

Usually $\epsilon_{n}$ lies in a limited range. Generating $\Gamma_{n}\left(t, x_{2}, x_{3}\right)=e^{i \cdot k \cdot t} \cdot \gamma_{n}\left(x_{2}, x_{3}\right)$ with large $\epsilon_{n}$ requires high energy. Thus we expect, by assuming $\epsilon_{n}$ relatively small, that the vibration frequencies of Class I (*-exact deformations) should locate around (1.1):

$$
\frac{|k|}{2 \pi} \approx \sqrt{\frac{\lambda+2 \mu}{\rho}} \cdot \frac{n}{2 l} \mathrm{~Hz} \quad(\text { Class I) }
$$

where $n$ is a positive integer.

Similarly we may deduce from Proposition 3.7 that some vibration frequencies of Class II (*-closed deformations) should appear around (1.2):

$$
\sqrt{\frac{\mu}{\rho}} \cdot \frac{n}{2 l} \mathrm{~Hz} \quad \text { (Class II). }
$$


On the other hand, we may infer from Proposition 3.8 that some other vibration frequencies of Class II (*-closed deformations) may appear at

$$
\sqrt{\frac{\mu}{\rho}} \cdot \frac{\sqrt{\epsilon}}{2 \pi} \mathrm{Hz} \quad \text { (Class II) }
$$

randomly.

\section{Acknowledgments}

The authors are indebted to the reviewer of this paper for providing insightful comments and important suggestions on an earlier version of this paper.

\section{References}

[1] D. N. Arnold, R. S. Falk and R. Winther, Finite element exterior calculus, homological techniques, and applications, Acta Numer. 15 (2006), 1-155.

[2] _ Finite element exterior calculus: From Hodge theory to numerical stability, Bull. Amer. Math. Soc. (N.S.) 47 (2010), no. 2, 281-354.

[3] M. Begout, Les problèmes liés au frottement élastomère-verre dans lautomobile, Ph.D. Thesis, Université Paul Sabatier de Toulouse, France, 1979.

[4] S. Berger, P. Ragot, J.-J. Sinou and E. Aubry, Model of chatter vibrations and stability analysis of a non-linear wiper system, Int. Rev. Mech. Eng. 2 (2008), no. 3, 349-356.

[5] G. Bódai and T. J. Goda, Friction force measurement at windscreen wiper/glass contact, Tribol. Lett. 45 (2012), no. 3, 515-523.

[6] _ Sliding friction of wiper blade: Measurement, FE modeling and mixed friction simulation, Tribol. Int. 70 (2014), 63-74.

[7] S. Cadirci, S. E. Ak, B. Selenbas and H. Gunes, Numerical and experimental investigation of wiper system performance at high speeds, J. Appl. Fluid Mech. 10 (2017), no. $3,861-870$.

[8] M. Chaichian, I. Merches and A. Tureanu, Mechanics, Springer-Verlag, Berlin, 2012.

[9] M. Chaichian, H. P. Rojas and A. Tureanu, Basic Concepts in Physics, SpringerVerlag, Berlin, 2014.

[10] T.-J. Chen and Y.-J. Hong, Mathematical formulas for the shear vibration frequencies of rubber wiper blade on convex windshield, to appear (2020). 
[11]__ Elasticity aspects of the shear vibration of rubber wiper blade on convex windshield, preprint (2020).

[12] M. Costabel, M. Dauge and S. Nicaise, Analytic regularity for linear elliptic systems in polygons and polyhedra, Math. Models Methods Appl. Sci. 22 (2012), no. 8, 1250015, $63 \mathrm{pp}$.

[13] M. Eastwood, A complex from linear elasticity, Rend. Circ. Mat. Palermo (2) Suppl. 63 (2000), 23-29.

[14] _ Ricci curvature and the mechanics of solids, Austral. Math. Soc. Gaz. 37 (2010), no. 4, 238-241.

[15] E. O. Ernst, The Boussinesq form of Saint Venant's principle: A demonstration, Math. Models Methods Appl. Sci. 10 (2000), no. 6, 863-875.

[16] T. Frankel, The Geometry of Physics: An introduction, Second edition, Cambridge University Press, Cambridge, 2004.

[17] D. Gilbarg and N. S. Trudinger, Elliptic Partial Differential Equations of Second Order, Classics in Mathematics, Springer-Verlag, Berlin, 2001.

[18] S. Goto, H. Takahashi and T. Oya, Clarification of the mechanism of wiper blade rubber squeal noise generation, JSAE Rev. 22 (2001), no. 1, 57-62.

[19] _ Investigation of wiper blade squeal noise reduction measures, SAE 2001 Noise \& Vibration Conference \& Exposition, (2001), Technical Paper 2001-01-1410, 6 pp.

[20] R. Grenouillat and C. Leblanc, Simulation of chatter vibrations for wiper systems, SAE 2002 World Congress \& Exhibition, (2002), Technical Paper 2002-01-1239, 8 pp.

[21] H. Haken and H. C. Wolf, Molecular Physics and Elements of Quantum Chemistry: Introduction to experiments and theory, Springer-Verlag, Berlin, 1995.

[22] Y.-J. Hong, Ruled manifolds with constant Hermitian scalar curvature, Math. Res. Lett. 5 (1998), no. 5, 657-673.

[23] _ Constant Hermitian scalar curvature equations on ruled manifolds, J. Differential Geom. 53 (1999), no. 3, 465-516.

[24] Harmonic maps into the moduli spaces of flat connections, Ann. Global Anal. Geom. 17 (1999), no. 5, 441-473.

[25] _ Gauge-fixing constant scalar curvature equations on ruled manifolds and the Futaki invariants, J. Differential Geom. 60 (2002), no. 3, 389-453. 
[26] Stability and existence of critical Kaehler metrics on ruled manifolds, J. Math. Soc. Japan 60 (2008), no. 1, 265-290.

[27] S. Klainerman and T. C. Sideris, On almost global existence for nonrelativistic wave equations in 3D, Comm. Pure Appl. Math. 49 (1996), no. 3, 307-321.

[28] G. Lancioni, S. Lenci and U. Galvanetto, Dynamics of windscreen wiper blades: Squeal noise, reversal noise and chattering, Internat. J. Non-Linear Mech. 80 (2016), 132143.

[29] D. Min, S. Jeong, H. H. Yoo, H. Kang and J. Park, Experimental investigation of vehicle wiper blade's squeal noise generation due to windscreen waviness, Tribol. Int. 80 (2014), 191-197.

[30] A. H. Muhr, Modeling the stress-strain behavior of rubber, Rubber Chem. Technol. 78 (2005), no. 3, 391-425.

[31] L. Nechak, S. Berger and E. Aubry, Prediction of random self friction-induced vibrations in uncertain dry friction systems ssing a multi-element generalized polynomial chaos approach, J. Vib. Acoust. 134 (2012), no. 4, 041015, 14 pp.

[32] S. Okura, T. Sekiguchi and T. Oya, Dynamic analysis of blade reversal behavior in a windshield wiper system, SAE 2000 World Congress, (2000), Technical Paper 200001-0127, 10 pp.

[33] T. Reddyhoff, O. Dobre, J. L. Rouzic, N.-A. Gotzen, H. Parton and D. Dini, Friction induced vibration in windscreen wiper contacts, J. Vib. Acoust. 137 (2015), no. 4, 041009, 7 pp.

[34] T. D. Rossing and N. H. Fletcher, Principles of Vibration and Sound, Springer-Verlag, New York, 2004.

[35] V. Serov, Fourier Series, Fourier Transform and Their Applications to Mathematical Physics, Applied Mathematical Sciences 197, Springer, Cham, 2017.

[36] T. C. Sideris, Nonresonance and global existence of prestressed nonlinear elastic waves, Ann. of Math. (2) 151 (2000), no. 2, 849-874.

[37] G. J. Stein, R. Zahoranský and P. Múčka, On dry friction modelling and simulation in kinematically excited oscillatory systems, J. Sound Vibration 311 (2008), no. 1-2, 74-96.

[38] P. R. Sundararajan, Physical Aspects of Polymer Self-assembly, John Wiley \& Sons, Hoboken, New Jersey, 2017. 
[39] W. Wang and S. Chen, Hyperelasticity, viscoelasticity, and nonlocal elasticity govern dynamic fracture in rubber, Phys. Rev. Lett. 95 (2005), no. 14, 144301, 4 pp.

Tsai-Jung Chen

Department of Vehicle Engineering, National Pingtung University of Science and Technology, Pingtung 912301, Taiwan

E-mail address: turtlechen29@hotmail.com

Ying-Ji Hong

Department of Mathematics, National Cheng-Kung University, Tainan 701401, Taiwan E-mail address: yjhong@mail.ncku.edu.tw 